(c) 2017. This manuscript version is made available under the Elsevier user license

\section{Introduction}

${ }^{*}$ Corresponding author

Email address: flponta@mtu.edu (Fernando L. Ponta)

Preprint submitted to Renewable Energy

\title{
Dynamic Aeroelastic Behavior of Wind Turbine Rotors in Rapid Pitch-Control Actions
}

\author{
Muraleekrishnan Menon ${ }^{\mathrm{a}}$, Fernando L. Ponta ${ }^{\mathrm{a}, *}$ \\ ${ }^{a}$ Department of Mechanical Engineering - Engineering Mechanics, Michigan Technological University, \\ Houghton, MI, 49931.
}

\begin{abstract}
Pitch control comprises a significant proportion of current wind turbine load-control approaches. Collective pitching is used in restricting the overall power generation at high winds, whereas individual pitching has the added advantage of mitigating cyclic loads that are detrimental in fatigue damage of the turbines. Currently, there are many studies on conventional pitching control that account for long-term variations in wind speeds and associated high loads, but a smaller number of studies in short-term pitch action.

The present study focuses on the use of rapid pitch control for handling short-term variations in wind conditions and load fluctuations within one cycle of rotation, with special attention to the prognosis of the aeroelastic response of the rotor. We use a numerical model capable of handling the complexities of the multi-physics dynamics of a wind turbine rotor. Based on a nonlinear adaptive ODE algorithm, it provides a natural way to integrate the various multi-physics aspects of wind turbine dynamics, including the control system and the coupled response of the aerodynamics and the structural deformations of the rotor. Results are presented for the case study of the NREL-5MW Reference Wind Turbine, and their significance for wind-turbine rotors in general is discussed.

Keywords:

Wind turbine, Rapid-action pitch-control, Innovative load-control
\end{abstract}

Wind is a major source of renewable energy that is clean and sustainable, and holds a promising share for the future of power generation. Recent studies conducted by European Wind Energy Association (EWEA) envision state-of-the-art turbines capable of generating $20 \mathrm{MW}$ with rotor diameters of $250 \mathrm{~m}$ [1]. The economies-of-scale factor indicates a trend towards increasing rotor sizes for reduced cost-of-energy in the wind power industry. This has led to various studies focusing on load mitigation control approaches [2, 3, 44]. Bianchi et al. [5] provides details on some of the most common load control methodologies. Some

February 1, 2017 
of these approaches currently being studied and implemented are stall control [6], pitch control [7, 8, 9], active stall control [10], passive control using aeroelastic devices [11], and active yaw control [12, 13]. One of the load-control approaches very widely implemented today and of interest to this study is pitch-control.

Control by pitch action comprises a significant proportion of present day turbine loadcontrol approaches. Most commercial turbines with pitch-control systems use a proportionalintegral collective pitching approach to prevent detrimental structural loads, and limit the power generated to their rated value during high winds [14]. The objective in these regimes of operation above nominal wind speeds is to reduce the aerodynamic torque by pitching to feather, and thus restrict the power generated. This ability to control the shaft torque through pitching is more beneficial as compared to stall-controlled turbines that result in high stochastic loads during operation. Collective pitching can be considered useful in restricting the overall power generation at high winds, whereas individual pitching has the added advantage of mitigating cyclic loads that are more detrimental in fatigue damage of the turbines [15, 16, 17, 18].

Fault Detection and Isolation (FDI) and Fault Tolerance Control (FTC) are other branches of wind-turbine control discipline that may benefit from the advanced aeroelastic predictive capabilities of the model we use in this study. Research interests in FDI and FTC, especially studies on fault detection and accommodation in pitch-control, have expanded in recent years, with several research works being published (see for example Gao et al. [19], Liu and Gao [20], Chen et al. [21], deBessa et al. [22], Odgaard et al. [23], Vidal et al. [24], Odgaard et al. [25], Odgaard and Johnson [26], Chen et al. [27], and Chaaban et al. [28] for more details).

There are many studies on conventional pitching control that account for long-term variations in wind speeds, and associated high loads, but a smaller number of studies in short-term pitch action. The present study focuses on the use of rapid pitch control for handling short-term variations in wind conditions and load fluctuations within one cycle of rotation, with special attention to the prognosis of the aeroelastic response of the rotor. This will provide valuable information to researchers specialized in the design of advanced control strategies to mitigate such effects.

Peaks of aerodynamic loading on the rotor produced by wind gusts, and instantaneous drops in wind loads due to tower interference are some examples of those short-term load fluctuations within the cycle of rotation. The periodicity involved in such loading as, for example, the one induced by tower interference is detrimental to the lifespan of the machine. The sporadic power fluctuations resulting from such scenarios affect the safe operation of the electro-mechanical devices connected to the turbine, and the electric grid in general. Designing control approaches to counter such swift and short fluctuations in loads is important for reliability in continued power production of turbines. Such approaches would need to target control actions implemented within the period of cyclic rotation, which in nominal operating conditions are in the order of a few seconds. Pitch mechanisms should hence be fast enough to counter cyclical variations like the ones associated with tower interference effects and/or the presence of gusts and turbulence. Pitching turbine blades of more than $100 \mathrm{~m}$ length and weighing 18 tons while operating under aerodynamic loading, and in such 
short spans, would demand high power of actuation, and may induce aero-elasto-inertial instabilities that may compromise the structural integrity of the rotor or drastically shorten its lifespan. This emphasizes the need for studying the aeroelastic response of wind turbine rotors for a better understanding of the scope and limits of such rapid pitch-control actions.

As an example, the dynamic response of the rotor of a National Renewable Energy Laboratory $5 \mathrm{MW}$ Reference Wind Turbine (NREL-5MW RWT) [29] undergoing fluctuating conditions due to tower interference is shown in figure 1. The NREL-5MW RWT constitutes a well known benchmark for a state-of-the-art turbine, and was used as a basis for designing cases of rapid pitch-control actuation for the present study. In the presence of the tower, measurable properties showed variations that affect the loads acting on the rotor and its resulting performance, for instance, in thrust and torque variations, and power deficits. Figure 1(a) shows the instantaneous power $P$ during a period of $5 \mathrm{~s}$, which is close to the period of rotation of the NREL-5MW RWT at its nominal operational conditions of $12.1 \mathrm{rpm}$. The $5 s$ span shows the three pulsations in the instantaneous power produced each time a blade crosses in front of the tower. Besides the negative effects on the instantaneous power, the pulsation produces a deficit in the averaged power compared with a rotor operating without tower interference, which are represented by the horizontal lines in figure 1(a).

This power pulsations result from the variations in driving torque produced in each cycle, which in turn is a consequence of fluctuations in the aerodynamic conditions at which the airfoil sections of the blade operate. In particular, changes in the angle of attack $\alpha$ crucially affect the lift and drag forces on each section along the blade span, and hence, the total torque, and thrust measured at the rotor's hub. As an example of the fluctuating aerodynamic conditions on the blade sections, figure 1(b) shows the angle of attack on the section located at $90 \%$ span from the root of the blade. This behavior is similar throughout the span, with the intensity of variation increasing towards the outer parts of the blade. For this reason, we have chosen $\alpha$ at this $90 \%$ span location as the focus of a substantial part of our study, being a critical aerodynamic parameter that characterizes the general behavior of the rotor in terms of its dynamic aeroelastic response.

In this study, we use the time-scale of those fluctuations induced by tower-interference as the basis for designing test cases to asses the dynamic response of the rotor. These test cases, presented in section 3 consist of rapid pitch-control actions exerted on similar time-scales.

\section{The Numerical Model}

Wind turbine blades are complicated structures that undergo cyclical rotating loads in stochastic wind conditions, which makes wind turbines a complex, non-linear dynamic system. Modeling wind turbines require many degrees of freedom (DOFs) to capture all features of the dynamic behavior of the rotor. The increasing size of the state-of-the-art rotor and these interlinking factors make wind-tunnel studies of next generation superturbines virtually impossible to extrapolate to the prototype scale, and thus prompt the need for full-scale studies using computer models. These models should also be capable of handling the complexities introduced by adding control systems that are coupled with aeroelastic modes of operation. 

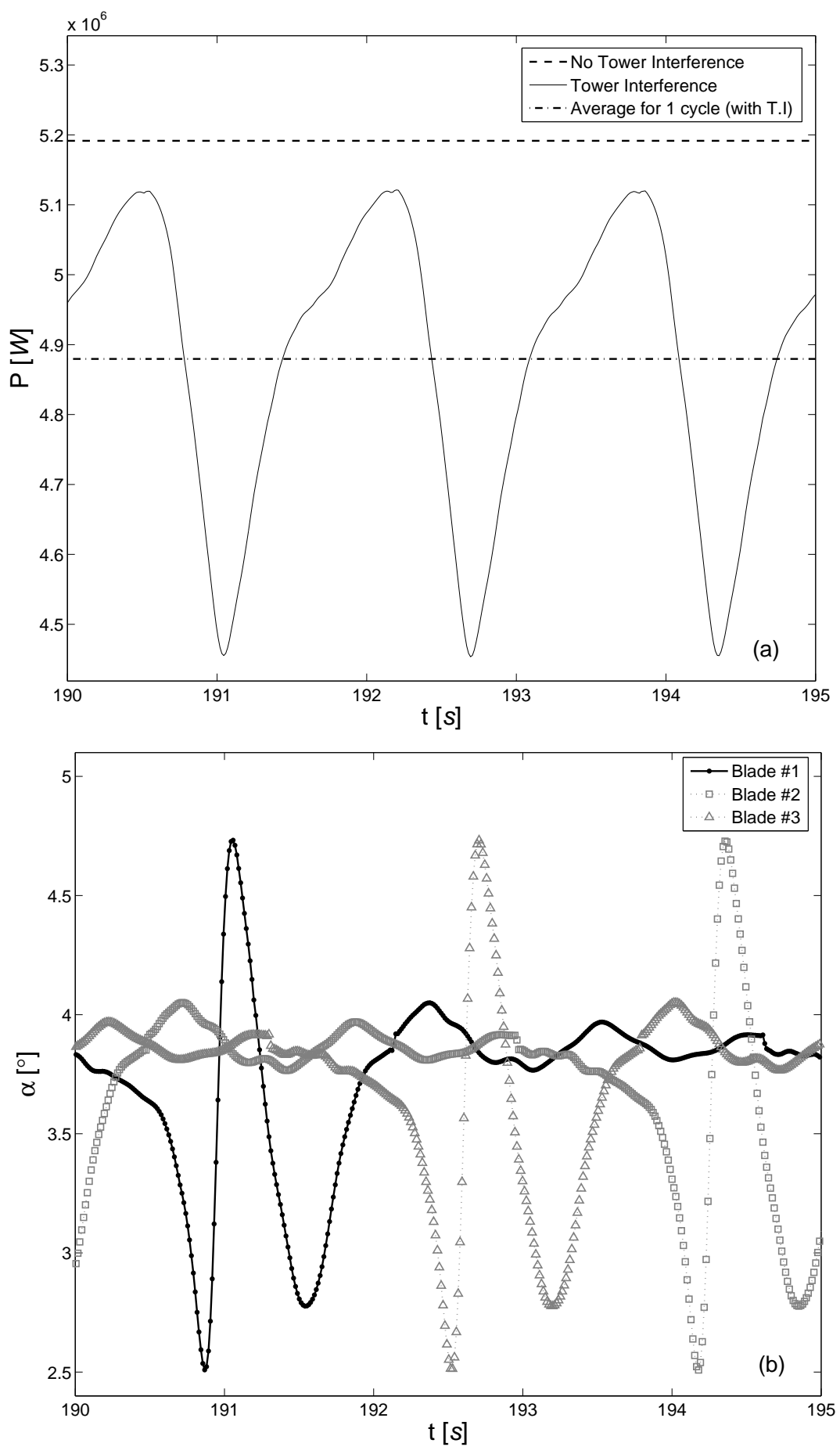

Figure 1: Rotor behavior due to tower interference - (a) power generated $P$, and (b) angle of attack $\alpha$, against time span on operation that includes one cycle of rotation. 
In this study, we use a novel numerical model capable of handling the aforementioned complexities. To make this paper self-contained, we provide a brief decription of the main features of the Dynamic Rotor Deformation - Blade Element Momentum (DRD-BEM) model [30]. The DRD-BEM model is capable of representing the coupled phenomena of the wind turbine using two advanced numerical schemes. First, a new implementation to model the structural response of heterogeneous composite blades [31], representing the complex modes of blade deformation while substantially reducing the computational effort. It uses the Generalized Timoshenko Beam Model (GTBM) approach, which is a dimensional reduction technique for complex beams originally proposed by Prof. Hodges and his collaborators [32, 33]. Second, the flow behavior is modeled using a novel aerodynamic technique based on the Blade Element Momentum (BEM) approach that allows representation of the instantaneous deformed configuration and the effects of rotor deformation into the computation of aerodynamic loads. This is achieved by the transformation of velocities acting at the rotor level through a series of orthogonal matrices projecting them on to the blade section, and in the same way re-projecting the forces acting at the blade section back to the rotor level where the interference is applied. These numerical schemes work in the context of a multi-physics solver called the Common Ordinary Differential Equation Framework (CODEF) [30], which also includes modules that model the dynamics of the control system and electromechanic devices on the drive-train.

For a detailed description of the implementation of the standard DRD-BEM model the reader is referred to Ponta et al. [30] and the references therein. Details of the structural model can be found in Otero and Ponta [31] and Ponta et al. [30]. The latter also includes results of the application of the DRD-BEM model to the analysis of vibrational modes of composite laminate wind-turbine blades, and results to validate the DRD-BEM model against the works of Jonkman et al. [29] and Xudong et al. [34].

\subsection{Blade structural model: Dimensional-reduction technique for beams}

The GTBM technique uses the same variables as the traditional Timoshenko beam theory but abandons the hypothesis that the beam sections remain planar after deformation. Instead, a 2-D finite-element mesh is used to interpolate the real warping of the deformed section, and a mathematical procedure is used to rewrite the strain energy of the 3-D body in terms of the classical 6 variables of the traditional 1-D Timoshenko theory for beams (i.e. the extensional strain, the two transverse shear strains, the torsional curvature, and the two bending curvatures). The complexity of the blade-section geometry and/or its material properties are reduced into a stiffness matrix for the equivalent 1-D beam problem and this can be done a priori in parallel for many blade cross sections. Elimination of the ad hoc kinematic assumptions of the traditional Timoshenko theory produces a fully populated $6 \times 6$ symmetric stiffness matrix for the 1-D beam, ensuring that the 6 modes of deformation are fully coupled. The non-linear, unsteady 1-D beam problem is then solved at each time step of the aeroelastic analysis through an advanced Ordinary Differential Equation (ODE) algorithm along a reference-line, $L$, that represents the axis of the beam on its original configuration. The procedure ensures that the strain energy of the reduced 1-D model is equivalent to the actual strain energy of the 3 -D structure in an asymptotic sense. 


\subsection{Rotor flow model: $D R D-B E M$}

The DRD-BEM is a numerical model representing the flow behavior on a wind turbine rotor based on the classical Blade Element Momentum model (BEM) [35]. Whereas the classical BEM approach misrepresents the aerodynamic forces due to a lack of complete representation of deformed cross-sections of the blade, the DRD-BEM model accounts for these deformations, and the resulting effects on aerodynamic loads itself. This is achieved through a series of orthogonal matrices that transform the velocities, and aerodynamic loads between coordinate systems representing specific aspects of the turbine. These aspects could be a pre-conformed manufacture setting such as a twist, cone angle, or pre-bending, or associated with a control action such as the pitching angle, yaw rotation, or rotor tilt.

We shall start from the velocity vector of the flow passing through an annular actuator disk aligned with the hub coordinate system, $h$. The components of this velocity vector are affected by an axial induction factor $a$, and a tangential induction factor $a^{\prime}$. These respectively represent the axial velocity deficit and the tangential velocity increase across the actuator disk. Then the velocity vector, $\mathbf{W}_{h}$, of the wind going through an annular actuator aligned with the hub coordinate system $h$ is given by,

$$
\mathbf{W}_{h}=\left[\begin{array}{l}
W_{\infty h_{x}}(1-a) \\
W_{\infty h_{y}}+\Omega r_{h} a^{\prime} \\
W_{\infty h_{z}}
\end{array}\right],
$$

where $\mathbf{W}_{\infty h}$ is the undisturbed wind velocity field referred to the hub coordinate system, $\Omega$ is the angular velocity of the rotor, and $r_{h}$ is the instantaneous radial distance of an annular disk traced by the rotating blade section (identified by $\delta l$ in the center panel in figure 2) on the rotor plane. This 3-D construction of $\mathbf{W}_{h}$ reflects how the stream-tubes associated with each blade element, aligned with $\mathbf{W}_{\infty h}$, gets deflected by the forces exerted on them by the annular actuators. A set of orthogonal matrices transform the wind velocity defined in a coordinate system aligned with the wind itself, $\mathbf{W}_{\infty \text { wind }}$, into the hub coordinate system, $\mathbf{W}_{\infty h}$, to account for cases like rotor tilt, and changes in yaw or wind direction. This unperturbed wind velocity in the hub coordinate system is obtained as:

$$
\mathbf{W}_{\infty h}=\left(\mathbf{C}_{\theta_{a z}} \mathbf{C}_{\theta_{t l t}} \mathbf{C}_{\Delta \theta_{\text {yaw }}} \mathbf{W}_{\infty \text { wind }}\right)
$$

where $\mathbf{C}_{\Delta \theta_{\text {yaw }}}$ accounts for the misalignment between wind direction and nacelle orientation due to yaw, $\mathbf{C}_{\theta_{t l t}}$ accounts for the misalignment due to rotor tilt through a rotation around the horizontal axis of the nacelle system, and the azimuthal orthogonal matrix $\mathbf{C}_{\theta_{a z}}$ transforms the wind velocity into the $h u b$ coordinate system $h$, by a rotation around the main shaft to the blade instantaneous position.

To compute the relative velocity of wind as seen by the instantaneous deformed blade section, $\mathbf{W}_{h}$ is projected through a few coordinate systems. This deformed configuration $\left(x_{l}, y_{l}, z_{l}\right)$ is defined along the deformed reference-line $l$ as depicted in the right panel of figure 2, and the velocity of wind in this reference-frame, $\mathbf{W}_{l}$ is given as:

$$
\mathbf{W}_{l}=\left(\mathbf{C}_{l L} \mathbf{C}_{L b} \mathbf{C}_{\theta_{p}} \mathbf{C}_{\theta_{c n}} \mathbf{W}_{h}\right)+\mathbf{v}_{s t r}+\mathbf{v}_{m e c h} .
$$




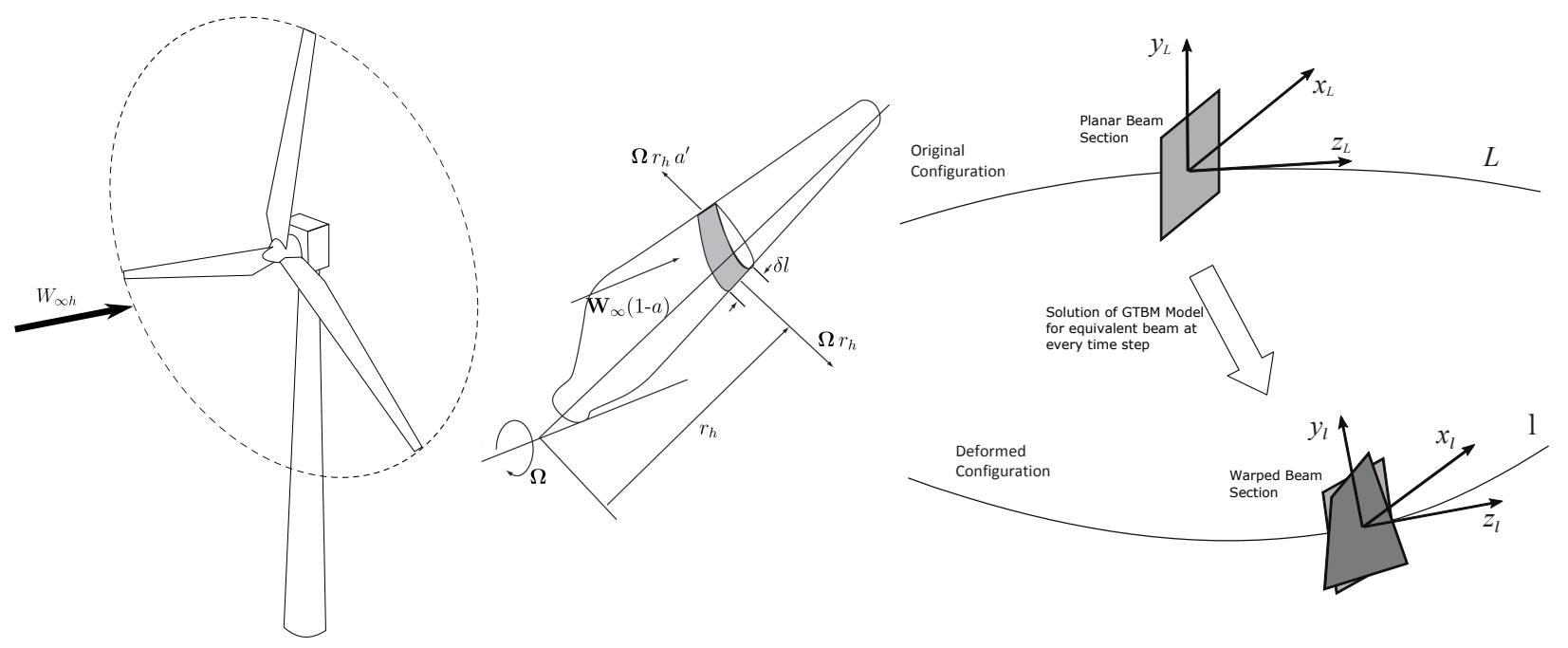

Figure 2: Schematic view of the dynamic generation of the annular actuator swept by a blade element (adaptation of a scheme present in Burton et al. 36] for classical BEM), with the schematic representation of Generalized Timoshenko Beam Model for a generic beam section. The latter shows the reference-lines, the beam sections, and the respective coordinate systems before and after deformation.

In equation 3, mathbf $C_{\theta_{c n}}$ denotes the transformation accounting for coning of the rotor, and mathbf $C_{\theta_{p}}$ performs a rotation around the pitching axis of the blade. These two transformations result in the blade coordinate system indicated by the subscript $b$ as per the IEC standards [37]. For a detailed description of the concept of coning rotors see [38, 39, 40]. Consequently, the orthogonal matrix $\mathbf{C}_{L b}$ transforms the velocity of wind from blade coordinate system $b$ to the non-deformed configuration system $L$, defined along the original reference line. And, the orthogonal matrix $\mathbf{C}_{l L}$ transforms from $L$ to instantaneous deformed configuration system $l$, which is obtained from the solution of kinematic aspects of the structural model explained in section 2.1. The intrinsic system $L$ is aligned to the blade section in the chord-normal, chord-wise, and span-wise directions, and represents the longitudinal axis of the beam in its original configuration, as depicted in the right panel of figure 2. In addition, $\mathbf{v}_{s t r}$ denotes the blade section vibrational velocities coming from the structural model, and $\mathbf{v}_{\text {mech }}$ is the velocity associated with the motion of the blade section due to combined action of mechanical devices such as yaw, pitch, and azimuthal rotation. Both these velocity vectors are already expressed in the $l$ system. As an example of all the transformations, the pitching rotation matrix is shown here,

$$
\mathbf{C}_{\theta_{\mathbf{p}}}=\left[\begin{array}{ccc}
\cos \left(-\theta_{p}\right) & \sin \left(-\theta_{p}\right) & 0 \\
-\sin \left(-\theta_{p}\right) & \cos \left(-\theta_{p}\right) & 0 \\
0 & 0 & 1
\end{array}\right],
$$

with the pitch angle $\theta_{p}=\theta_{p_{0}}+\theta_{p_{c t r l}}$, where $\theta_{p_{0}}$ is a fixed angle set up as an initial operational parameter, and $\theta_{p_{c t r l}}$ is the variation of pitching angle exerted by the control system. 


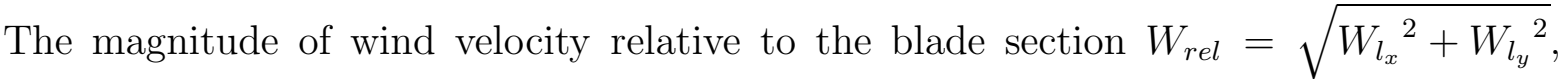
and angle of attack $\alpha$ can be used to compute aerodynamic lift and drag forces per unit span length, denoted by $\delta \mathbf{F}_{r e l}$. These forces are then projected back to the $h$ coordinate system, which are obtained as $\delta \mathbf{F}_{h}=\mathbf{C}_{\theta_{c n}}^{T} \mathbf{C}_{\theta_{p}}^{T} \mathbf{C}_{L b}^{T} \mathbf{C}_{l L}^{T} \mathbf{C}_{\text {Lthal }} \mathbf{d} \mathbf{F}_{\text {rel }} \delta l$, where $\mathbf{C}_{\text {Lthal }}$ projects the lift and drag forces onto the chord-normal and chord-wise directions, aligned with the coordinates of $l$.

A major step in this model is equating the forces obtained from the Blade Element Theory to the change of momentum defined as per the Momentum Theory. Components of $\boldsymbol{\delta} \mathbf{F}_{h}$ are hence equated to the rate of change of momentum through the corresponding annular actuator. The component normal to the actuator, $\delta F_{h_{x}}$ is equated to the change in momentum on $W_{\infty h_{x}}$, which is associated with the axial interference factor $a$ (see expression 11), and the tangential component $\delta F_{h_{y}}$ is equated to the corresponding momentum change associated with tangential induction factor $a^{\prime}$.

The final step in the process of transitioning between the aeroelastic and structural modes is to compute the distributed loads and moments acting on the blade structure per unit span length. These forces expressed in the deformed configuration system $l$ constitute both aerodynamic forces and gravitational loads and are required as inputs for the structural model. After determination of the induction factors, the process from equation 1 through equation 3 is repeated to compute the aerodynamic forces on each blade section, however, this time in the $l$ system. That is, $\mathbf{d F}_{l}=\mathbf{C}_{\text {Lthal }} \mathbf{d F}_{r e l}$, whose first two components will give the chord-normal and chord-wise aerodynamic loads. The aerodynamic moment on the airfoil section per unit span length acting around the first axis of $l$ is computed at the blade sections as

$$
d M_{\text {aer }}=\frac{1}{2} \rho C_{m} W_{\text {rel }}^{2} c^{2}
$$

where $C_{m}$ is the aerodynamic pitch coefficient for the corresponding angle of attack, $\alpha$.

\subsection{The Common ODE Framework (CODEF)}

Hitherto, we have seen how our structural model will interact with our aerodynamic model providing a comparable level of description in order to make full use of the advanced capabilities of both models. This notion of integral dynamic multi-physics modeling through an ODE solution in time could be extended to include other aspects that greatly affect the dynamics of the rotor and the overall performance of the wind-turbine, like the response of the control-system and/or the turbine's electromechanical devices.

As it was mentioned above, the equations of motion for the 1-D finite-element problem of the equivalent beam are solved using a nonlinear adaptive ODE solver. This type of solver is based on variable-timestep/variable-order ODE algorithms that check the solution by monitoring the local truncation error at every timestep, improving the efficiency and ensuring the stability of the time-marching scheme. The differential equations modeling the dynamics of the control system and electromechanical devices may be added to the general ODE system, with the control and the electromechanical dynamics modifying the boundary conditions for the aeroelastic solution and vice-versa. The use of a nonlinear 


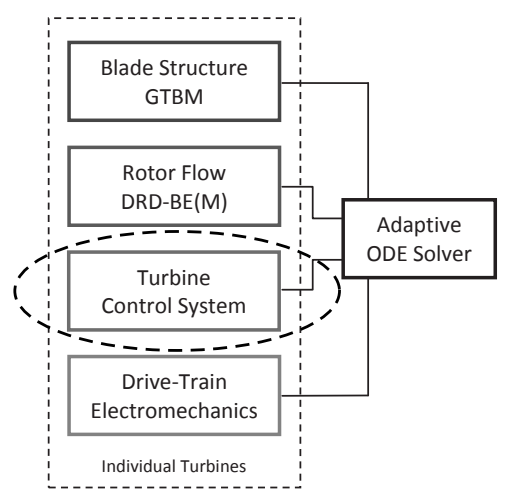

Figure 3: Flow-chart diagram of the Common ODE Framework.

adaptive ODE algorithm as a common framework provides a natural way of integrating the solution of all the multi-physics aspects of the problem. Figure 3 shows a flow-chart diagram of this global scheme indicating the interrelation between the different modules. These modules may be treated individually, interfacing with the common ODE routine. Contrary to a monolithic approach, this modular design of our multi-physics model substantially simplifies further development of the code by the improvement and/or expansion of each submodel independently. This makes possible the simultaneous analysis of the aeroelastic problem, together with any innovative control strategy involving all physical aspects of the turbine dynamics (mechanical and electrical), by means of an integral computationallyefficient solution through a self-adaptive algorithm. Moreover, it opens the door in the future for an interconnection of the dynamics of individual turbines into an integral simulation of their collective dynamics within a wind-farm, including all physical aspects of turbine-toturbine interaction: aerodynamic, electrical, and collective control at farm-level.

\section{Numerical Experimentation}

Wind turbine blades are complex structures akin to twisted beams, with airfoil-profile cross sections that vary in their twist angle, sectional shape, and chord length, from the root to the tip. As a result, the aerodynamic loads and the associated structural loads are different throughout the span of the blade. On the other hand, pitching as a control action alters the alignment of the blade with the wind at the root, imposing the same angular change along the entire blade. Due to the changes in shape and inflow conditions, the aerodynamic loads acting on the various sections along the span are quantitatively different. However, these changes in aerodynamic behavior throughout the blade span follow a similar qualitative pattern, resulting in a global change in behavior that, when integrated along the whole span, produces a consistent control action. Thus, studying the aeroelastic behavior at one particular section is representative of the qualitative dynamics of the entire blade and provides insights into the main physical mechanisms responsible for that dynamics. In any wind-turbine blade, the sections closer to the tip are designed to be more aerodynamically efficient and contribute more to the driving torque which contributes to power production. Measuring aeroelastic observable properties in these span sections is an effective way to 


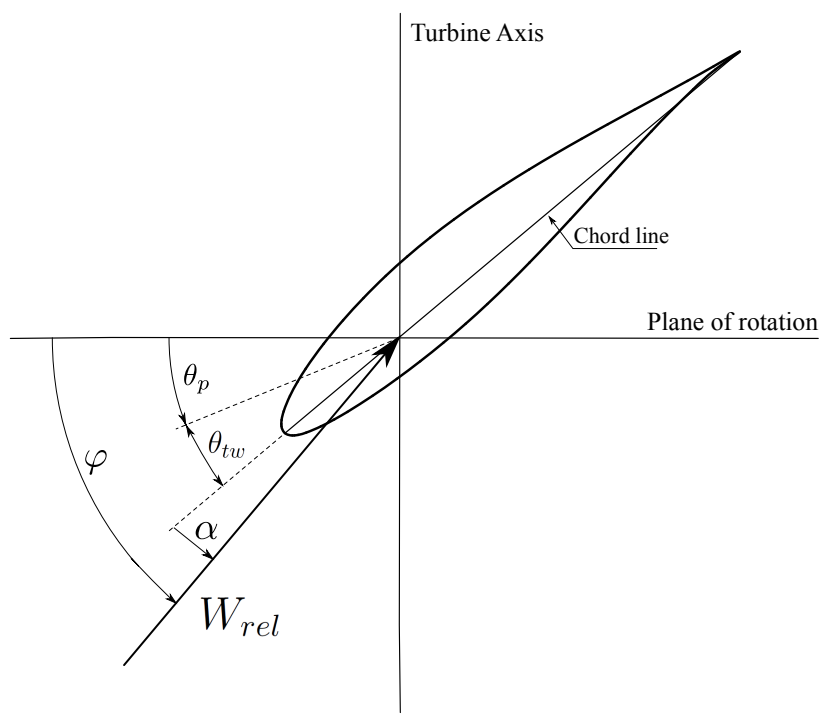

Figure 4: Schematic of a 2-D airfoil section of the blade, showing angles relevant to pitch control action.

understand the rotor response to control-pitch action. Selecting the $90 \%$ span section for analysis is convenient as it promises to show intense vibrational and deformational effects (as closest possible to the tip), but still avoid any tip effects. This effectively takes into account the combined dynamics of the structural and aerodynamic effects on the blade. The aerodynamic forces acting on the blade directly depend on the angle of attack, $\alpha$, which makes it a key observable for assessing the blade loads. This property also reflects the characteristics of the rotor resulting from both geometrical alterations due to dynamic deformations and the kinematics changes of the flow due to the operation of the machine itself as a mechanism. Hence, in a complex dynamic system like this with coupled aeroelastic modes of operation, the angle of attack could be seen as a measurable property that serves as a nexus between the geometrical/kinematic aspect of the problem and the dynamic one.

The NREL-5MW RWT used in this study is a 3-bladed horizontal-axis wind turbine, with a rotor diameter of $126 \mathrm{~m}$, and a rotor coning of $2.5^{\circ}$. The rated output power for the turbine is $5 \mathrm{MW}$, when operating at a rotational speed of $12.1 \mathrm{rpm}$ in a wind speed of $11.4 \mathrm{~m} / \mathrm{s}$. These nominal operating conditions provides the primary setting for the results presented in sections 3.1 and 3.2, which were computed via the virtual test environment of the CODEF multi-physics model (see section 2). We use these results to asses the main features that characterize the turbine response to rapid pitch-control actions. These experiments are later extended in section 3.3 to operational conditions at wind speeds above the nominal.

Figure 4 shows a schematic of a 2-D airfoil blade section, showing the angles relevant to pitch control action: the angle of attack $\alpha$, and its relationship with the angle of pitch $\theta_{p}$, the angle of incidence of the flow $\phi$, and the twist angle of the section $\theta_{t w}$. The twist angle depends on structural properties such as the design twist angle of the section in the blade's original, un-deformed configuration $\theta_{L b_{z}}$, and the instantaneous deformational twist $\theta_{l L_{z}}$. These properties vary dynamically when pitch control actions are exerted at the root of the blade, according to its complex non-linear aeroelastic dynamics. This results in a change in the angle of attack $\alpha$, which does not mimic exactly the change in pitching by 
a given control action, $\theta_{p_{c t r l}}$. As it was mentioned above, $\alpha$ is an essential parameter that defines the aerodynamic forces acting on the section, and thus is the target of the control action itself. Assessing the actual relationship between the control action represented by $\theta_{p_{c t r l}}$ and the corresponding change in $\alpha$ is the first aspect that we shall analyze.

To determine the time-span on which a typical rapid-pitch control action should be implemented, we use as a starting point the observations made in section 1 about the rotor response to tower interference. The objective is to study the behavior of aerodynamic observable properties (in particular, $\alpha$ ), in a hypothetical attempt to mitigate the aerodynamics effects of tower interference, or any other fluctuations in conditions with similarly short time-scales. As it was mentioned in section 1, the time-scale of those tower-interference fluctuations are used as the basis for designing test cases with defined pitch-control actions exerted on similar periods of time. The basic test control action is a positive step change in pitch angle, $\theta_{p_{c t r l}}$, applied as a collective pitching of all the blades simultaneously, and is completed within one rotation cycle. The simulation of the turbine operation continues for a few more cycles of rotation, as needed for the aeroelastic transient modes of the rotor to develop into a stable state of operation.

\subsection{Aeroelastic blade response to pitching control}

Pitching action as a load-control mechanism during operation works by inducing adjustments to the angle of attack on the blade sections. The latter also depends on the the instantaneous orientation the incident flow at different sections along the span, which is affected by the aerodynamic interference of the rotor on the incoming wind. The standard blade used in the NREL-5MW is a flexible one, that is, it is capable of deforming under operation. This makes it difficult to discriminate how much of the change in $\alpha$ could be attributed to the pitching action, how much to the change in the angle of the incident flow $\phi$, and how much to the change in twist $\theta_{t w}$ due to the torsional deformation of the blade. In order to isolate the contribution from the change in the incident flow due to aerodynamic interference, a hypothetical hyper-stiff blade was designed, with a structural stiffness $10^{3}$ times that of the standard blade. This does not alter the aerodynamic shape of the standard blade or its inertial properties, but reduces deformation to a negligible value. By ruling out deflection and torsion, the hyper-stiff blade shows the magnitude of the contribution of aerodynamic interference to the change in $\alpha$ for a certain fixed change in pitching angle $\theta_{p}$.

At its nominal operation conditions, the NREL-5MW has an initial pitch angle, $\theta_{p_{0}}$ of $0^{\circ}$. The control-action was imposed starting at $20 s$ of stable operation, which is approximately equivalent to 4 cycles of rotation. Figure 5 shows the evolution of $\alpha$ on the section at $90 \%$ of the span, when a series of prescribed pitch-control actions of a determined angle were imposed. A positive pitching action results in a drop in the angle of attack from its original value which is not identical to the value of $\theta_{p_{c t r l}}$ imposed; and it can also be seen that, with different angles of pitching, the proportional change in the angle of attack $\Delta \alpha$ with respect to the control action imposed is not the same. This variation in the $\Delta \alpha / \theta_{p_{c t r l}}$ response for the hyper-stiff blade is exclusively related with the changes in aerodynamic interference.

The next step is to study the dynamic response of the NREL-5MW rotor using the standard blade. Even though its general behavior is similar to the one observed for the 


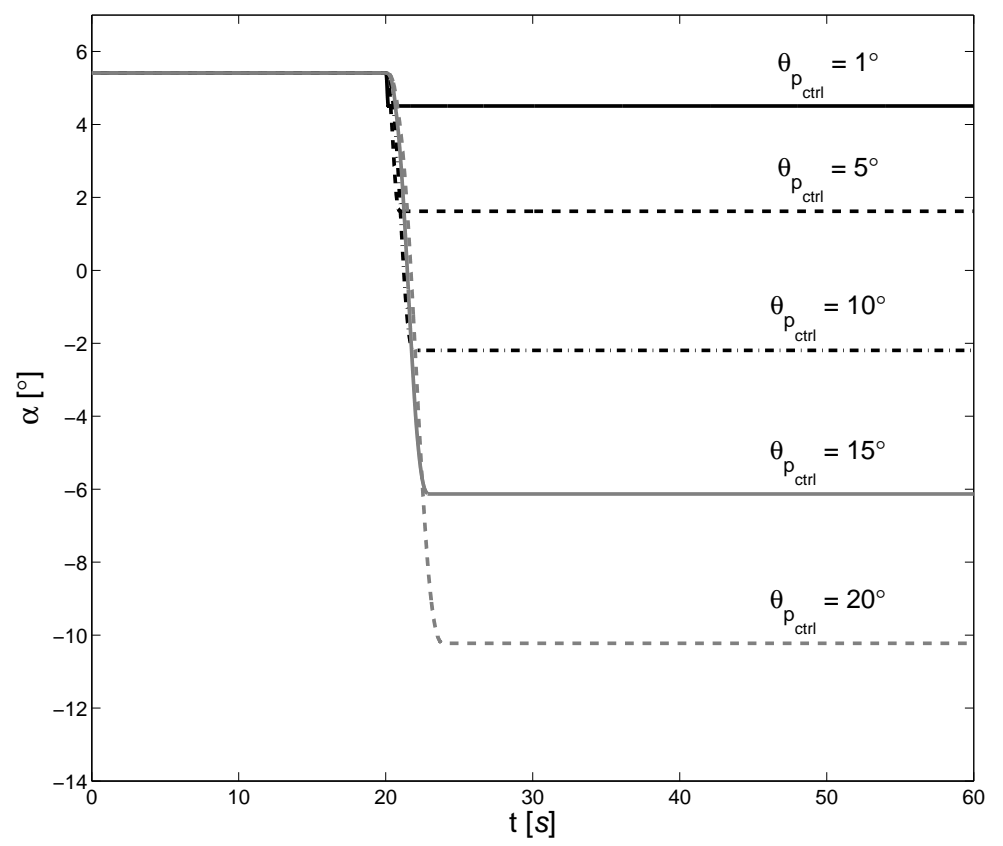

Figure 5: Time evolution of $\alpha$ on the section at $90 \%$ of the span for a series of prescribed pitch-control actions of a determined angle $\theta_{p_{c t r l}}$.

hyper-stiff blade, now the deformational effects are present, and the aeroelastic interaction introduces new aspects in the dynamic response of the rotor that depend not only on the amount of the pitch-control action, but also on how fast this action is applied. An example of this could be seen in figure 6, where the change in $\alpha$ is shown for the case of a fixed control action of $5^{\circ}$ of pitch applied during different time-spans, i.e., involving different angular acceleration of the pitching action $\Gamma_{\theta_{p_{c t r l}}}$.

The first aspect that we notice is that there is a systematic consistency in the variation of $\alpha$ achieved when the aeroelastic response stabilizes after a transient period (i.e., the value of the final plateau in the different plots), and that this variation only depends on the angle of pitching, $\theta_{p_{c t r l} l}$. That is, the end result of the control action seems to be independent of the speed at which pitching takes place, like in the case of the hyper-stiff blade, even though the transient behavior now depends strongly on the pitching acceleration. The value of the final plateau in the $\alpha$ signal is different than the one observed in the hyper-stiff blade for a similar $\theta_{p_{c t r l}}$, due to the contribution to the twist by the torsional deformation. In order to verify the behavior observed in the section at $90 \%$ of the blade span, the same type of analysis was repeated for other sections located at different positions along the external (i.e., the aerodynamically active) portion of the blade. Figure 7 shows the $\Delta \alpha / \theta_{p_{c t r l}}$ relation in function of the pitch-control action $\theta_{p_{c t r l}}$ at three different locations along the blade span: $90 \%, 70 \%$, and $50 \%$, which indicates a consistent behavior. The dotted lines show the curves for the hyper-stiff blade, and the continuous lines the case of the standard blade, which serves as a comparison between a purely aerodynamic vs. a fully aeroelastic response.

The second characteristic aspect that could be observed in the dynamic response of the rotor with standard blades, shown in Figure [6, is the presence of oscillations in the transient 

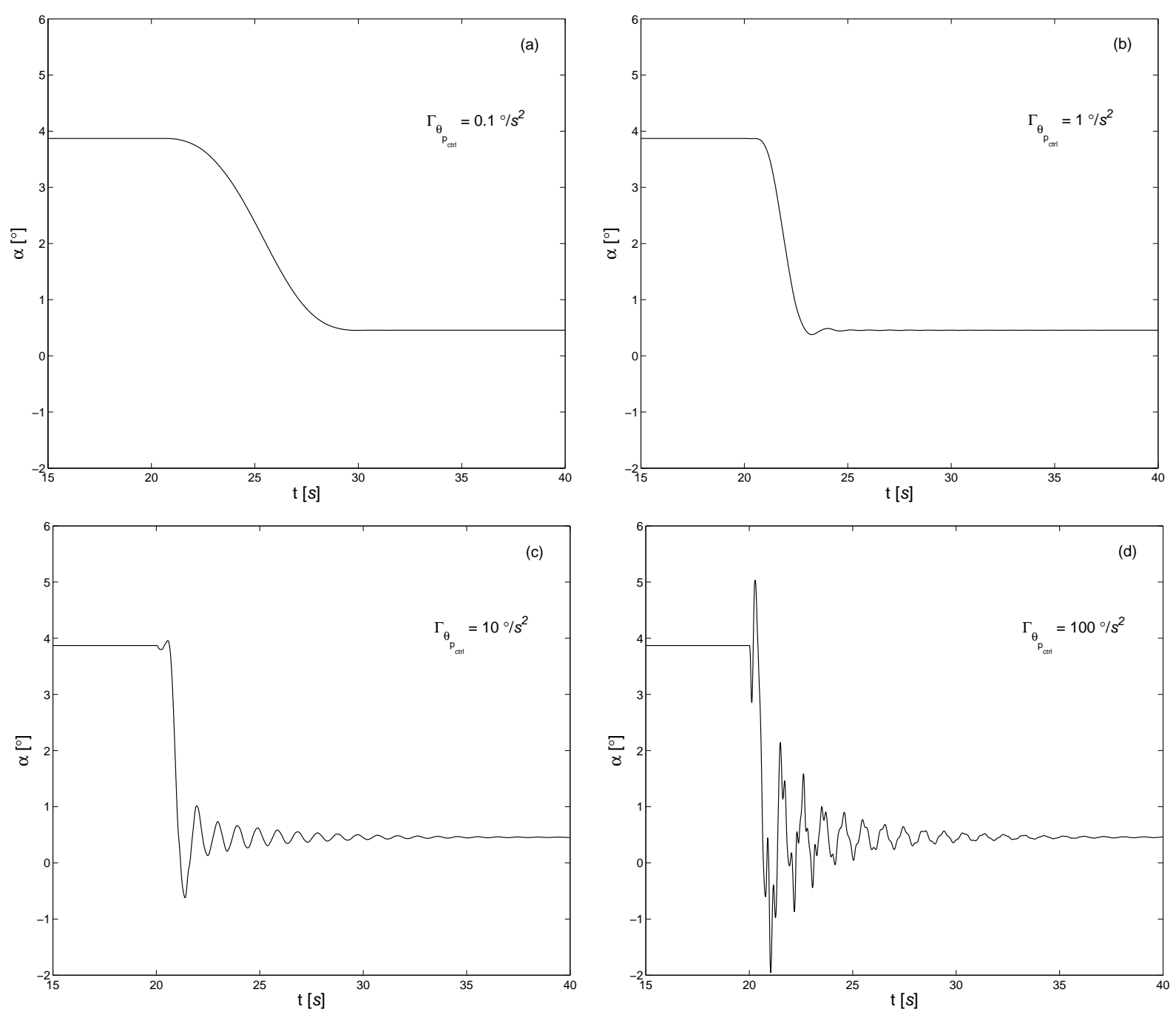

Figure 6: Change in angle of attack, $\Delta \alpha$ for a control pitch action of $\theta_{p_{c t r l}}=5^{\circ}$ with different accelerations of pitching, $\Gamma_{\theta_{p_{c t r l}}}$; (a) $0.1 \% / s^{2}$, (b) $1 \% / s^{2}$, (c) $10 \% / s^{2}$, (d) $100 \% s^{2}$ 


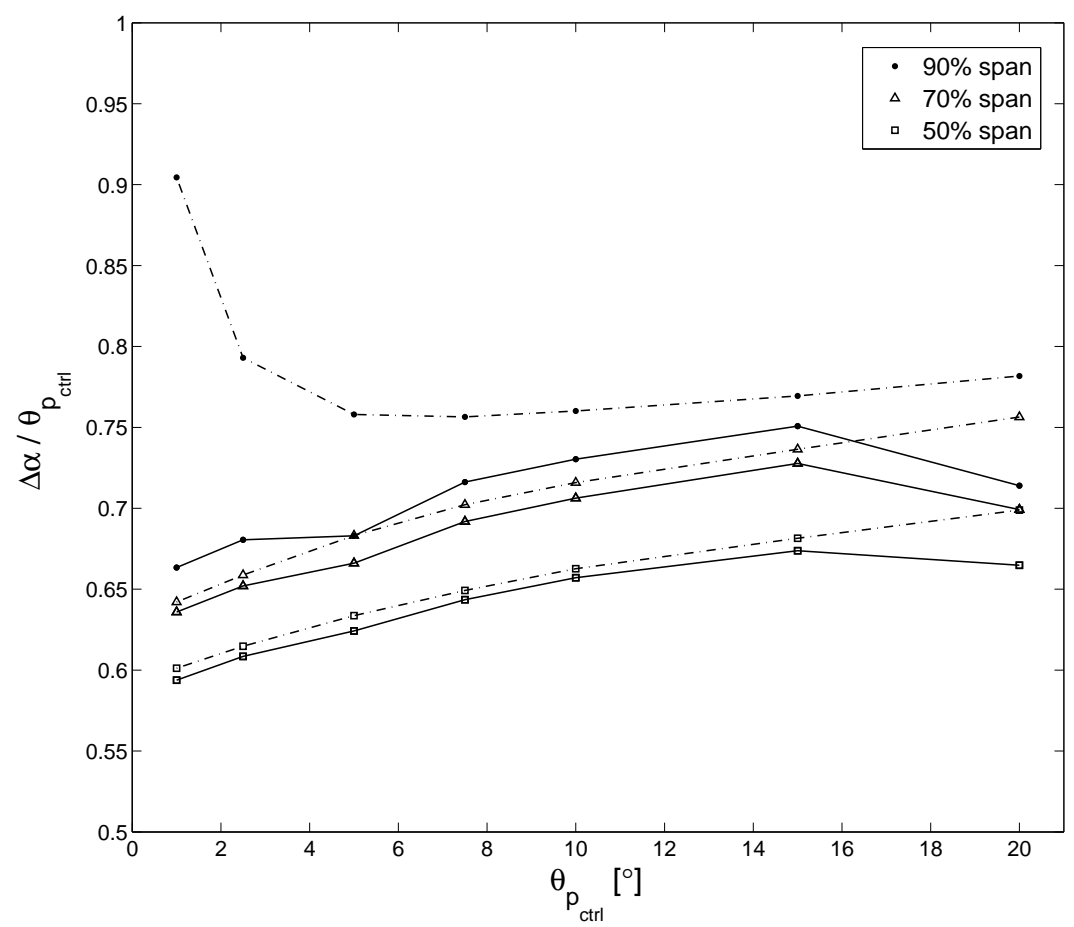

Figure 7: The $\Delta \alpha / \theta_{p_{c t r l}}$ relation in function of the pitch-control action $\theta_{p_{c t r l}}$ at different locations along the span. The dotted lines show the curves for the hyper-stiff blade, and the continuous lines the case of the standard blade.

period before a constant value of $\alpha$ is achieved, whose nature depends on the value of pitching acceleration. The panels in figure [ 6 illustrate four distinctive types of behavior observed at different values of $\Gamma_{\theta_{p_{c t r l}}}$. First, at low values of acceleration like in figure 6(a), the transient evolution of $\alpha$ is completed without oscillations, similar to the case of the hyper-stiff blade. When the level of acceleration is increased, a second type of behavior could be observed in figure 6 (b) where noticeable, but still small oscillations appear in the aeroelastic response after pitching. This is followed by a range of acceleration values where a consistent behavior of periodic oscillations of a single fundamental frequency, which is depicted in the example shown in figure 6 (c). Finally, figure [6 (d) shows the evolution of $\alpha$ at higher values of acceleration where a more complex oscillatory behavior could be observed, indicating the presence of richer spectrum of frequencies.

\subsection{Oscillatory blade response induced by rapid pitching action}

As shown in the previous section (see figure 6), for a given value of the pitch action $\theta_{p_{c t r l}}$, the evolution in the transient period before the blade reaches a stable configuration exhibits four distinctive states in terms of its qualitative behavior, whose nature depends on the value of pitching acceleration. In this section, we focus on the main aspects of the oscillatory response observed at higher pitch-acceleration values, e.g. the states depicted in figures 6(c) and 6(d). First, we identified the threshold in pitch acceleration that leads to the appearance of single-frequency oscillations and the threshold at which a multi-frequency content is observed, i.e. the states whose spectra are depicted in figures 8 (a) and 8 (b) respec- 

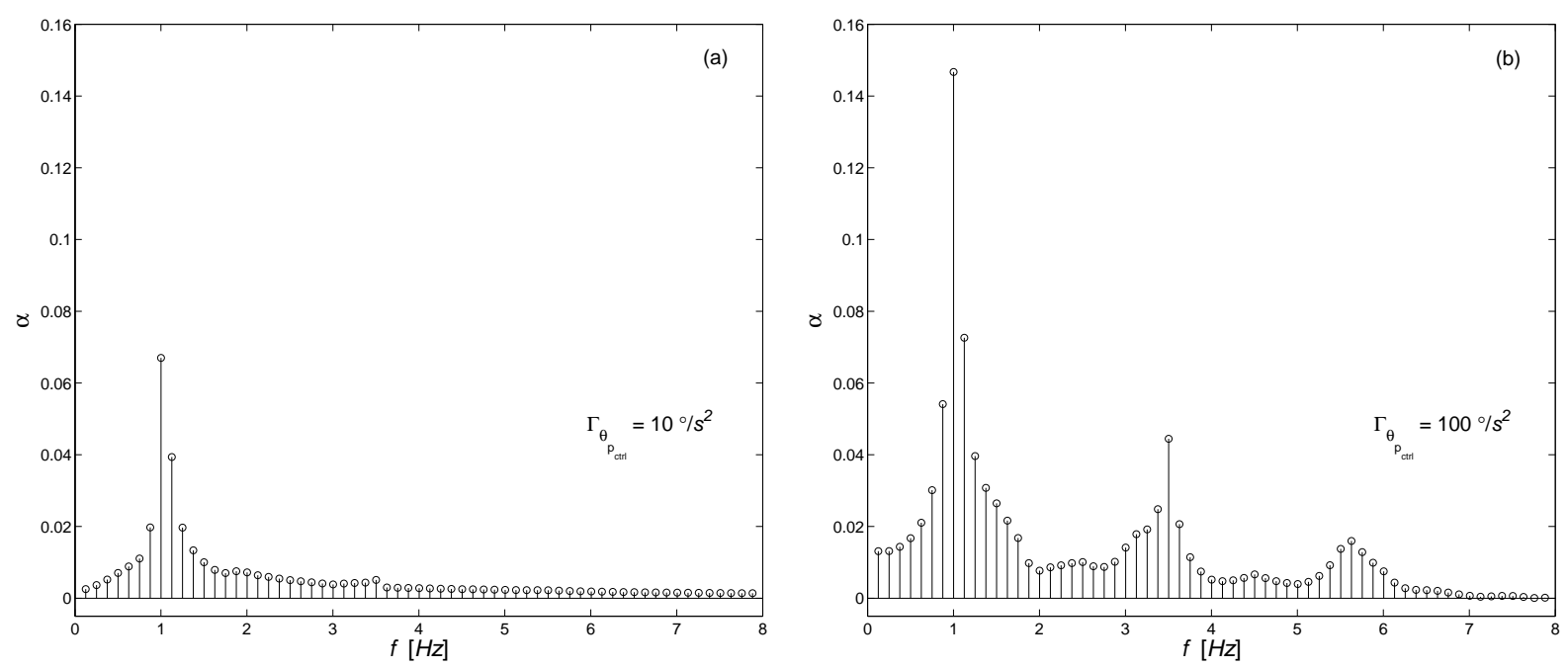

Figure 8: Frequency content in the aeroelastic response of the rotor for a pitch actuation of $\theta_{p_{c t r l}}=5^{\circ}$ at two different accelerations of pitching; (a): acceleration of $10^{\circ} / \mathrm{s}^{2},(\mathrm{~b})$ : acceleration of $100^{\circ} / \mathrm{s}^{2}$. These spectra correspond respectively to cases (c) and (d) in figure 6 .

tively. The values of these transitional thresholds depend on the amount of the pitch action $\theta_{p_{c t r l}}$, and are plotted in figure 9 for a pitching range from $\theta_{p_{c t r l}}=1^{\circ}$ to $\theta_{p_{c t r l}}=15^{\circ}$. The light-gray shaded region below lower threshold indicates pitching conditions at accelerations low enough to result in an aeroelastic response with minor or negligible oscillations. The shaded region above the upper threshold indicates pitching accelerations at which a multifrequency content is present. As a way of example, figure 9 also includes two markers $(\nabla)$ showing the locus of the cases for $\Gamma_{\theta_{p_{c t r l}}}=10^{\circ} / \mathrm{s}^{2}$ and $\Gamma_{\theta_{p_{c t r l}}}=100^{\circ} / \mathrm{s}^{2}$, which correspond to the spectra shown in figure 8, and the evolutions shown in figures 6(c) and 6(d).

The region in between the two thresholds, shown in white in figure 9, is characterized by a consistent oscillatory behavior with a single fundamental frequency which has a consistent value slightly above $1 \mathrm{~Hz}$, with a slight dependence on the pitch actuation angle, ranging from a minimum of $0.993 \mathrm{~Hz}$ for $\theta_{p_{c t r l}}=1^{\circ}$, to a maximum of $1.071 \mathrm{~Hz}$ for $\theta_{p_{c t r l}}=20^{\circ}$. The oscillations also exhibit a consistent damping that depends only on the value of $\theta_{p_{c t r l}}$, examples of which can be observed in the time evolutions of $\alpha$ shown in figure 10 for four different pitching angles from $\theta_{p_{c t r l}}=1^{\circ}$ to $\theta_{p_{c t r l}}=20^{\circ}$. This exclusive dependency on an aerodynamic variable such as $\theta_{p_{c t r l}}$ indicates that the damping is predominantly aeroelastic in nature, more than purely due to material properties of the blade structure. The four different cases plotted in figure 10 illustrate the change in damping as $\theta_{p_{c t r l}}$ increases, going progressively from rapid attenuation to actual amplification of the aeroelastic vibrations, with a zero damping situation (where the amplitude remains practically constant) occurring at about $\theta_{p_{c t r l}}=16.25^{\circ}$. Figure 11 shows the value of the logarithmic decrement $\delta$, characterizing the damping at the nominal wind speed of $11.4 \mathrm{~m} / \mathrm{s}$ for different values of $\theta_{p_{c t r l}}$, and the solid line connecting the markers represents the best-fit cubic curve to the data. This qualitative behavior of the oscillations, in terms of frequency and damping observed at nominal wind speed is also consistently exhibited at higher values of the operational wind speed, which will be the focus of section 3.3. 


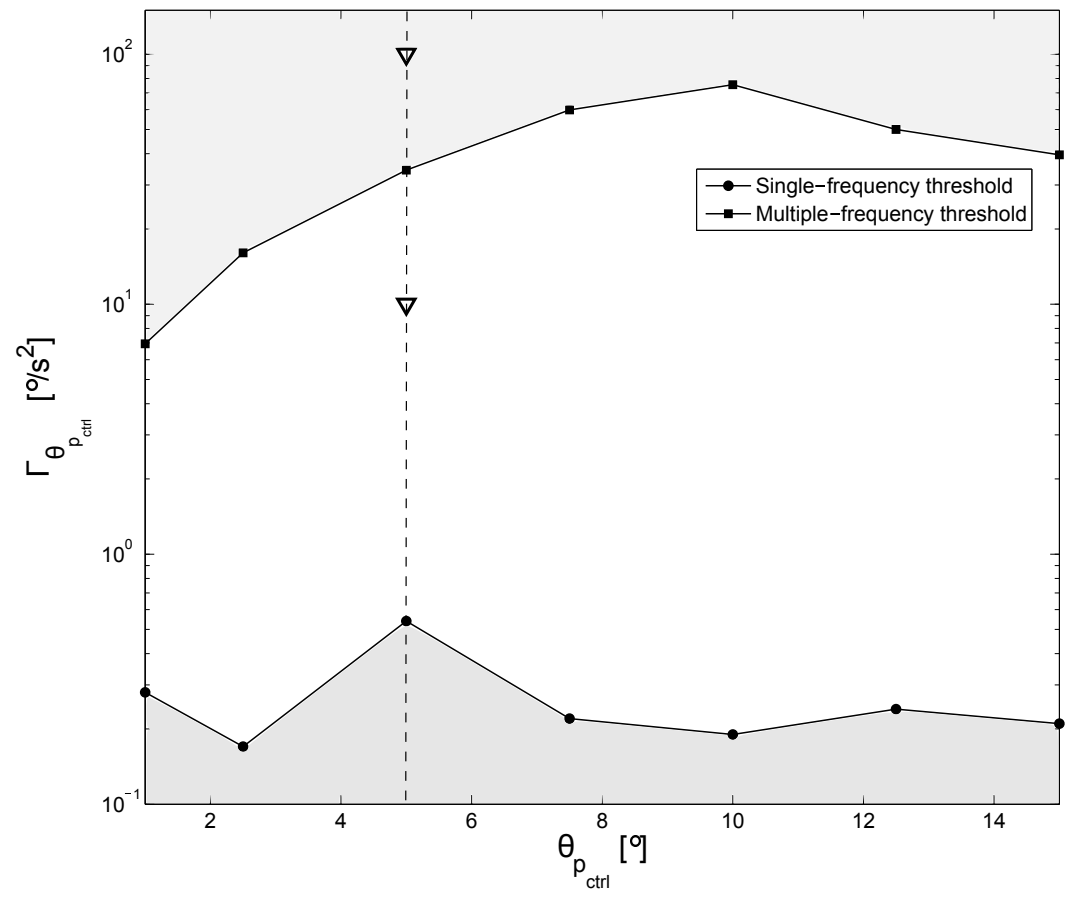

Figure 9: Thresholds on acceleration of pitching actuation for the appearance of single-frequency periodic oscillations, and for the appearance of multiple-frequency content. Markers $(\nabla)$ indicate the locus of cases for $\Gamma_{\theta_{p_{c t r l}}}=10^{\circ} / s^{2}$, and $100^{\circ} / s^{2}$, at $\theta_{p_{c t r l}}=5^{\circ}$, corresponding to the examples shown in figures 6 (c) and 6(d), and the frequency spectra shown in figure 8 .

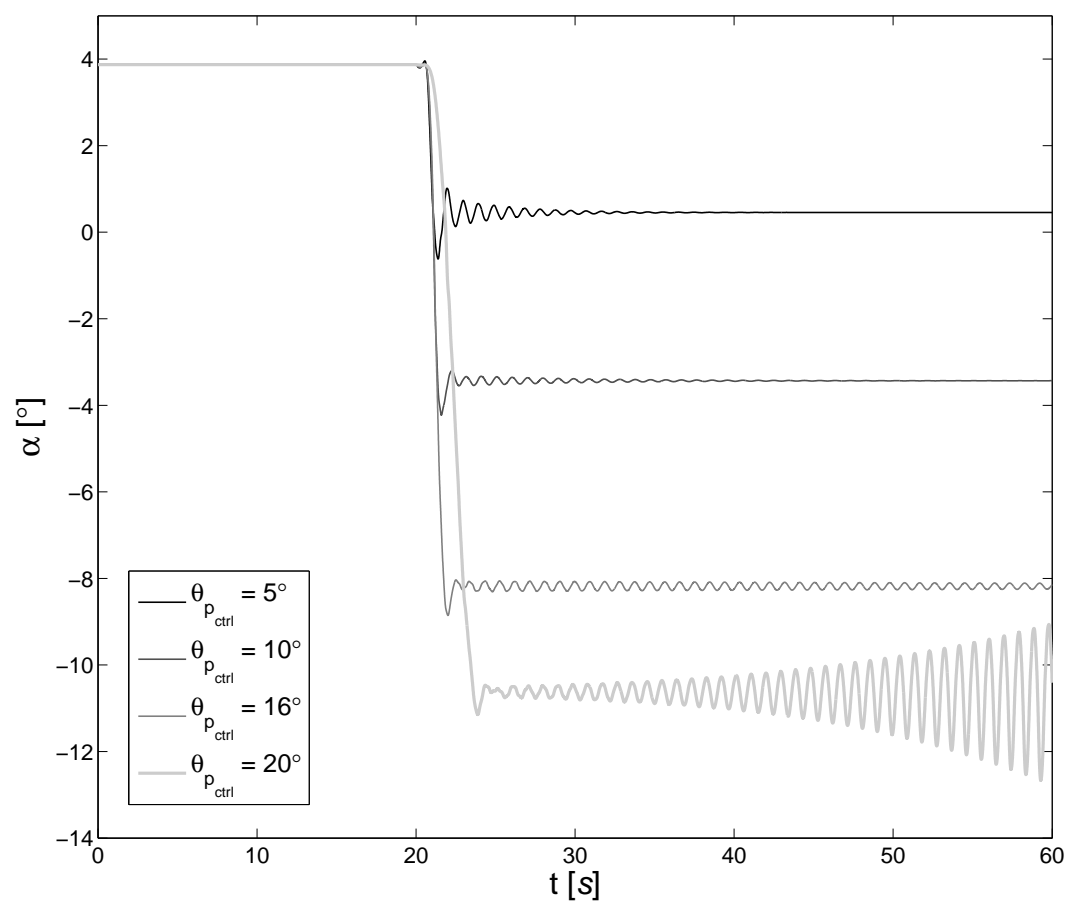

Figure 10: Examples of time evolution of the angle of attack $\alpha$ for four different pitching angles, $\theta_{p_{c t r l}}$. 


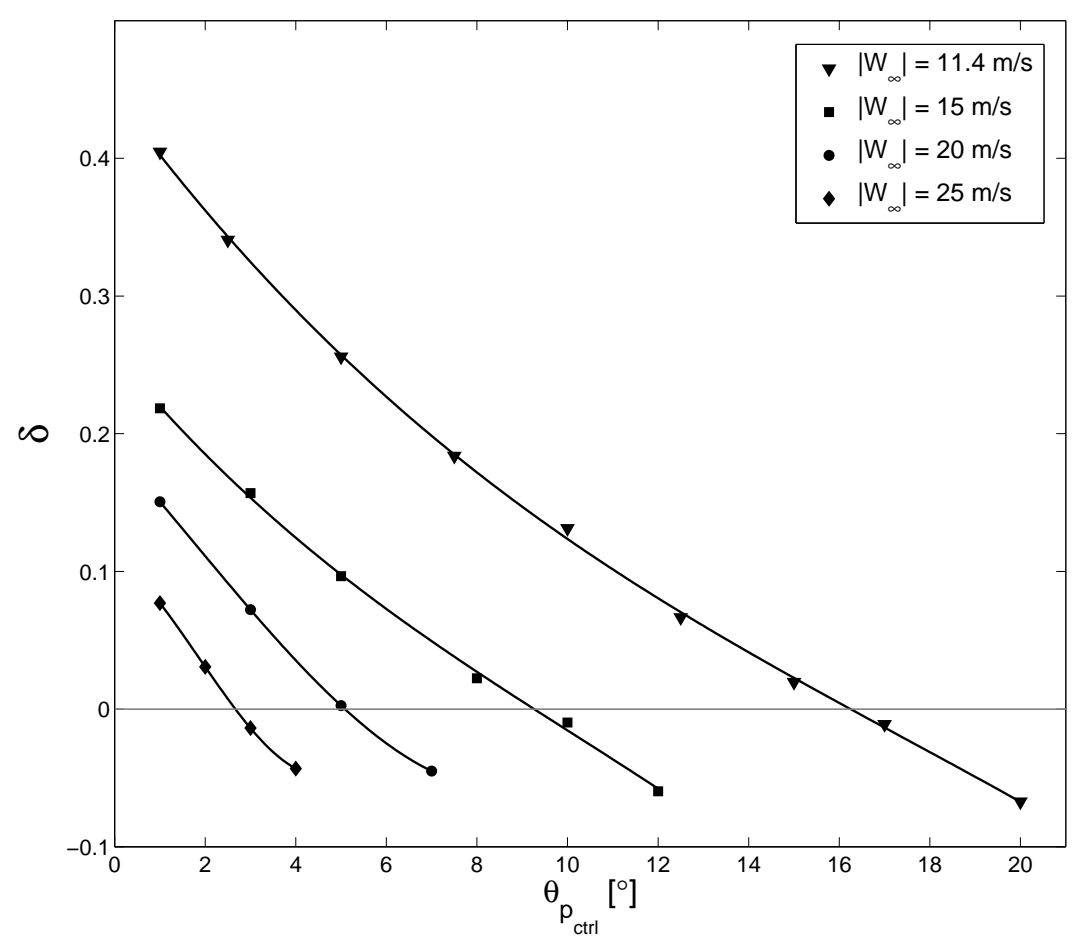

Figure 11: Logarithmic decrement $\delta$, characterizing the damping at the nominal wind speed of $11.4 \mathrm{~m} / \mathrm{s}$ for different values of $\theta_{p_{c t r l}}$ (the solid line connecting the markers represents the best-fit cubic curve to the data). Also shown is the damping behavior for three wind speeds above nominal, $W_{\infty}=15,20$, and $25 \mathrm{~m} / \mathrm{s}$.

Verification studies conducted on global rotor parameters during the rapid pitch actuation shows consistency with previous observations about blade-section parameters. The output power $\mathrm{P}$, rotor thrust $\mathrm{T}$, and blade-tip displacement, $u_{h_{x_{t i p}}}$, were assessed in similar test conditions for rapid pitching. A positive pitching (feathering) produced drop in output power, and the final value showed dependency only on the amount of pitch actuation, $\theta_{p_{\text {ctr }} l}$. This behavior is qualitatively identical to the one observed in the time evolution of $\alpha$ for the airfoil section at $90 \%$ of the blade span (see figure 6), which support our initial selection of $\alpha$ at this location as a significant parameter to study. Accordingly, blade-tip displacement and rotor thrust also show similar behavior on their time evolution.

\subsection{Rotor response to rapid pitching for wind speeds above the nominal}

Figure 11 also shows the results for $\delta$ at three different wind speeds above the nominal: $15 \mathrm{~m} / \mathrm{s}, 20 \mathrm{~m} / \mathrm{s}$, and $25 \mathrm{~m} / \mathrm{s}$, which covers the entire upper range of operation for the NREL$5 \mathrm{MW}$ turbine until its cut-off wind speed. The objective of this part of our study was to assess the consistency in rotor response to rapid control actions for operational conditions at which the initial pitch $\theta_{p_{0}}$ is no longer zero as it was in the case of the nominal speed. That is, the rapid control action adds to an initial existing pitch which is required to maintain the output power at its rated level for wind speeds above nominal. For the examples shown in figure 11, the rotor blades operate at a pitch angle of $\theta_{p_{0}}=9.27^{\circ}$ for wind speed of $15 \mathrm{~m} / \mathrm{s}$, $\theta_{p_{0}}=16.2^{\circ}$ for $20 \mathrm{~m} / \mathrm{s}$, and $\theta_{p_{0}}=21.8^{\circ}$ for $25 \mathrm{~m} / \mathrm{s}$ (see Table 2 from Ponta et al. [30]).

The behavior observed at these higher wind conditions is qualitatively equivalent to the 
nominal case. That is, final values of $\alpha$ only depend on the corresponding angles of pitch actuation, and the characteristics of the oscillatory motion during the transient period until a stable value of $\alpha$ is achieved depends on the pitching acceleration. The damping behavior is also consistent, with a dependence only on the pitch actuation angle. In figure 11 we could see that the value of $\delta$ for the same $\theta_{p_{c t r l}}$ decreases as wind speed increases. This is a consequence of the change in geometrical configuration of the rotor, which is subjected to a higher deformation when operating at higher wind speeds. Angle of the rapid pitching action at which a zero-damping situation occurs $\left(\theta_{p_{c t r l}(\delta=0)}\right)$ also reduces as the wind speed increases. This is expected due to the fact that the pitching action, $\theta_{p_{c t r l}}$, starts not from zero as in the nominal case, but from a required initial pitch $\left(\theta_{p_{0}}\right)$ that increases with wind speeds beyond the nominal value. However, the total value of pitching angle at which zerodamping occurs $\left(\theta_{p_{(\delta=0)}}=\theta_{p_{0}}+\theta_{\left.p_{c t r l(\delta=0)}\right)}\right)$ increases. Figure 12 shows the curves for both $\theta_{p_{c t r l}(\delta=0)}$ and $\theta_{p_{(\delta=0)}}$ vs wind speed, markers correspond to the same four wind speeds cases shown in figure 11, and solid lines show their respective best-fit curves based on a cubic polynomial fitting.

The fundamental frequencies for these oscillations in cases of wind speeds above nominal also show a consistent behavior with the value slightly above $1 \mathrm{~Hz}$, with a slight dependence on the pitch actuation angle, $\theta_{p_{c t r l}}$. For wind speed of $15 \mathrm{~m} / \mathrm{s}$, the value of frequency ranges from a minimum of $1.012 \mathrm{~Hz}$ for $\theta_{p_{c t r l}}=1^{\circ}$ to a maximum of $1.06 \mathrm{~Hz}$ for $\theta_{p_{c t r l}}=12^{\circ}$. For wind speed of $20 \mathrm{~m} / \mathrm{s}$, the value of frequency ranges from a minimum of $1.031 \mathrm{~Hz}$ for $\theta_{p_{c t r l}}=1^{\circ}$ to a maximum of $1.063 \mathrm{~Hz}$ for $\theta_{p_{c t r l}}=7^{\circ}$. For wind speed of $25 \mathrm{~m} / \mathrm{s}$, the value of frequency ranges from a minimum of $1.045 \mathrm{~Hz}$ for $\theta_{p_{c t r l}}=1^{\circ}$ to a maximum of $1.063 \mathrm{~Hz}$ for $\theta_{p_{c t r l}}=4^{\circ}$.

\section{Conclusions and outlook for further work}

The nonlinear adaptive ODE algorithm used in this work provides a natural way to integrate the various multi-physics aspects of wind turbine dynamics. The capacity of CODEF framework (described in section 2.3) to reflect the coupled aeroelastic response is critical to properly capture essential elements in the prognosis of turbine response against rapid control actions. A simple structural analysis of the vibrational response of the blades would not show this phenomenon, as the aerodynamic component of the coupled problem is missing. A typical example of this was noted in section [3.2, where we referred to the aeroelastic nature of the damping of blade oscillations.

This aeroelastic aspect of the damping behavior could be critical for the safety of the turbine. The curves of total pitch angle for zero damping, $\theta_{p_{(\delta=0)}}$, in function of wind speed shown in figure 11 implies a well defined safety limit in the amount of pitching that could be applied when operating at a certain wind speed. Regardless of how slow the pitching action occurs, if the machine continues operating at a condition with damping less than zero, any oscillation created would be amplified indefinitely until it compromises the structural integrity of the rotor. Hence, for multi-megawatt turbines like the NREL-5MW RWT asserting those aspects could prove critical in determining to what extent, and how 


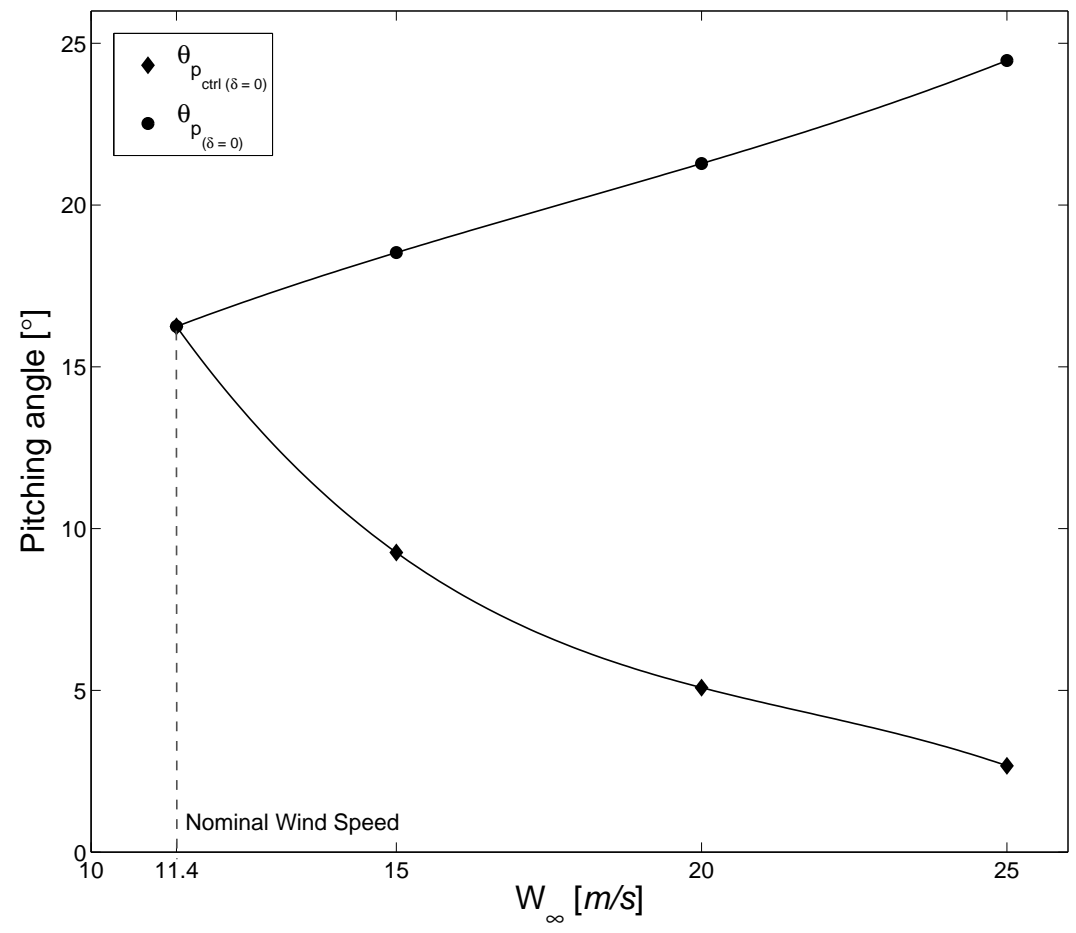

Figure 12: $\theta_{p_{c t r l(\delta=0)}}$ and $\theta_{p_{(\delta=0)}}$ vs wind speed. Markers correspond to the same four wind speed cases shown in figure 11] and solid lines show their respective best-fit curves based on a cubic polynomial fitting.

rapidly, pitching actions could be exerted without compromising the stable operation of the rotor, its safety, and/or its operational life due to the accumulation of fatigue effects.

The various aspects of the turbine response discussed in section 3 indicate direct relations between the dynamic response in the aeroelastic behavior of the rotor, and a quantitative measure of the control-action given, i.e. the amount of the pitch actuation angle and the characteristic acceleration of the rapid pitching process. Here, we shall elaborate on some fundamental aspects regarding the physical mechanisms underlying the observed rotor response.

The first aspect to note is that variations in the angle of attack, $\Delta \alpha$ result from the cumulative effect of changes in other properties, such as the angle of relative wind, $\phi$ (which is essentially aerodynamic, and depends on rotor interference), the torsional deformation, $\theta_{l L_{z}}$ (which is essentially structural, and depends on rotor deformation), and the pitching angle itself (which is essentially mechanical, and depends on the control system). For the case of the hyper-stiff blade presented in section 3.1, torsional deformations are negligible, and the twist angle at a certain section along the blade span, $\theta_{t w}$ remains constant at its design value, $\theta_{L b_{z}}$, in the original non-deformed configuration (see figure 44). Thus, any difference between the $\Delta \alpha$ and the intended change by the control action $\theta_{p_{c t r l}}$ is due to the effect of aerodynamic interference inducing a change in the angle of incidence $\Delta \phi$. This means that the value of $\Delta \alpha$ is given by the subtraction of $\theta_{p_{c t r l}}$ from $\Delta \phi$. When the same control action is applied to the standard blade, not only is torsional deformation present, but also the airfoil sections along the span are no longer perpendicular to the pitching axis due to the curvature of the axis of the blade (i.e. the reference-line, L, see section 2) because of bending. The 

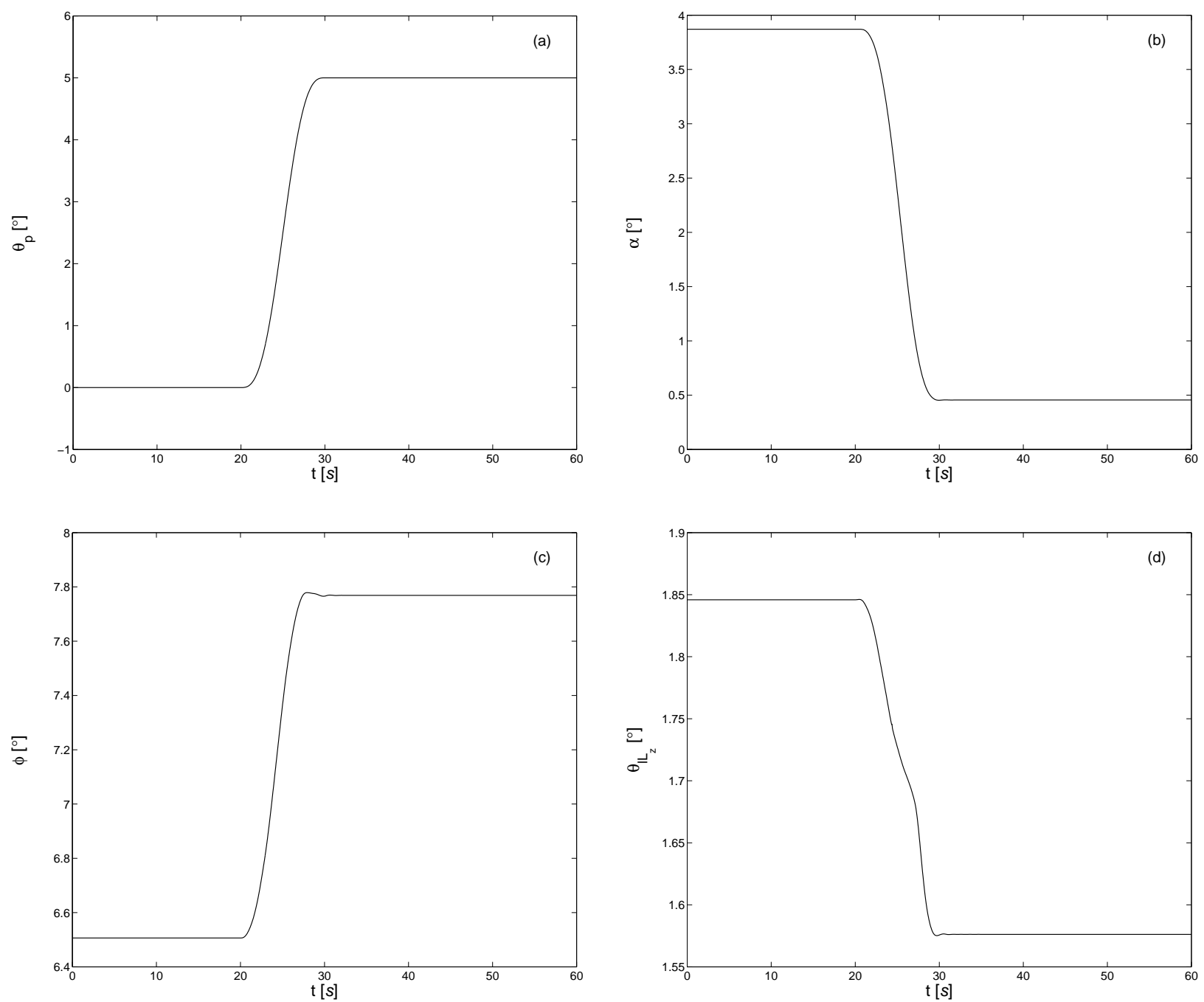

Figure 13: Time evolution of aerodynamic and structural properties of standard rotor operation at nominal conditions, for a control pitch actuation of $\theta_{p_{c t r l}}=5^{\circ},(\mathrm{a})$ angle of pitching $\theta_{p}$, (b) angle of attack $\alpha$, (c) angle of relative wind $\phi,(\mathrm{d})$ angle of torsional deformation $\theta_{l L_{z}}$.

presence of this misalignment due to bending means that the simple subtraction of $\theta_{p_{c t r l}}$ from $\Delta \phi$ will no longer coincide with $\Delta \alpha$ even if the addition of the torsional deformation, $\theta_{l L_{z}}$ is considered. Nevertheless, it is still possible to analyze the factors that affect $\Delta \alpha$, as it is still primarily determined by the combined effect of the same parameters: $\Delta \phi, \theta_{l L_{z}}$, and $\theta_{p_{c t r l}}$. Figure 13 shows an example of the simultaneous time evolution of the four parameters for the case of a control action of $\theta_{p_{c t r l}}=5^{\circ}$ in the standard rotor operating at nominal conditions (here, we selected a case where pitching actuation was applied at an acceleration low enough to preclude oscillations, in order to facilitate observation of the relative evolution of each parameter).

The second aspect to note in terms of the physical mechanisms underlying the rotor dynamics is related to the origins of the frequency content in the oscillatory behavior on the transient period after a rapid control action is applied, which could be observed in the spectra shown for $\alpha$ in figure 8. A logical assumption is that the fundamental frequency of oscillation 

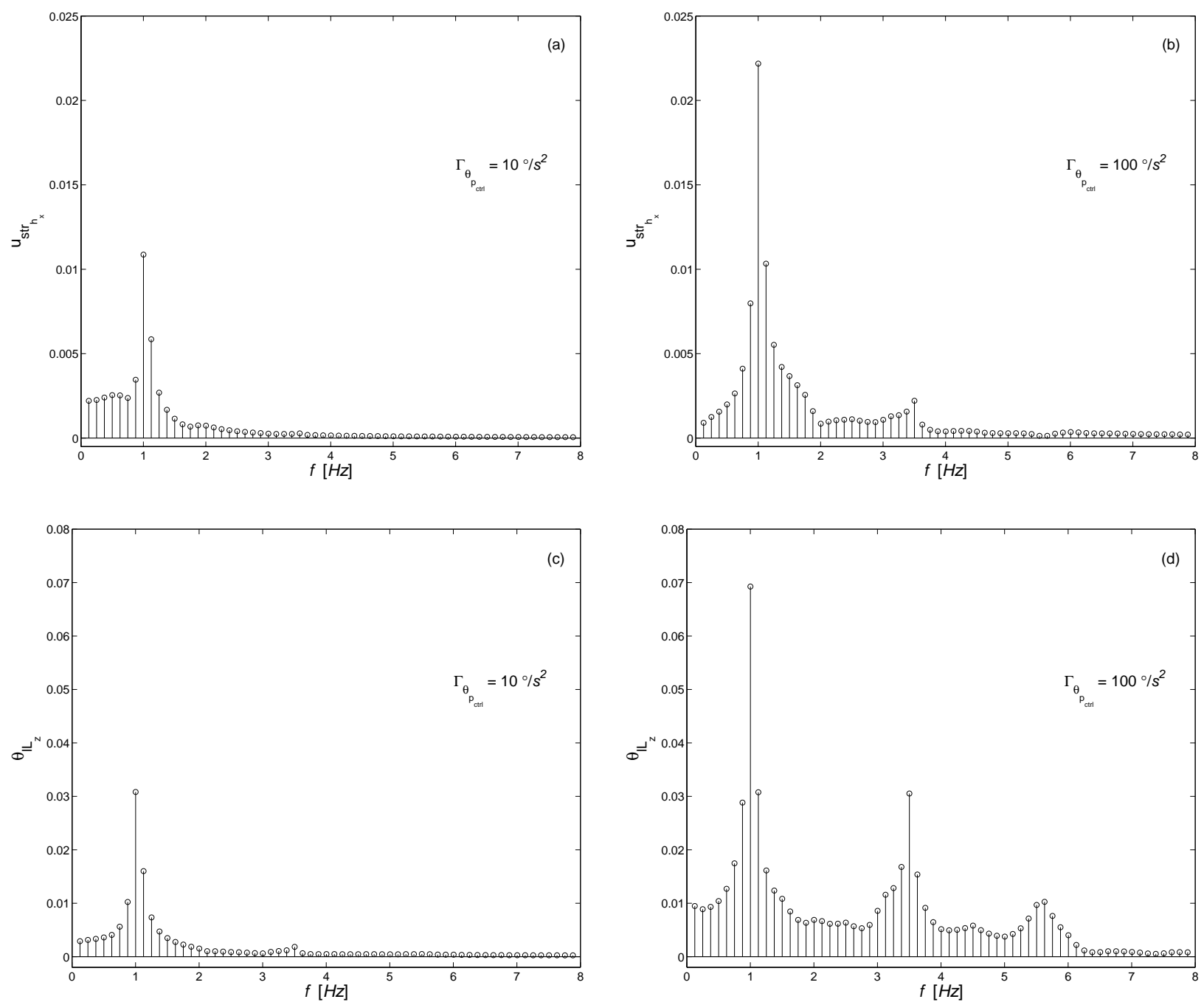

Figure 14: Spectra of axial displacement, $u_{s t r_{h_{x}}}$, and the torsional deformation, $\theta_{l L_{z}}$ for a pitch actuation of $\theta_{p_{\text {ctrl }}}=5^{\circ}$ on the standard rotor in nominal operational conditions at the $90 \%$ span blade section; (a) and (c) for a pitching acceleration, $\Gamma_{\theta_{p_{c t r l}}}=10^{\circ} / s^{2}$, (b) and (d) for $\Gamma_{\theta_{p_{c t r l}}}=100^{\circ} / s^{2}$. These spectra correspond to the respective cases shown for $\alpha$ in figure 8 ,

comes primarily from the most important mode of deformation of the blades, which is the flapwise bending mode. Flapwise oscillation introduces out-of-rotor-plane vibrational velocities on the motion of the blade section, affecting the direction of the incident flow and its angle $\phi$, which in turn affects $\alpha$. Besides, the GTBM structural model used in the CODEF is capable of capturing the coupled flexo-torsional modes of deformation of the blade. Hence, the oscillating bending deformation of the flapwise mode is also reflected into the frequency content of the oscillations in the torsional angle of deformation, $\theta_{l L_{z}}$, which in turn also affects $\alpha$. This coupled nature of the aeroelastic dynamics makes it challenging to totally isolate the origin of the different frequencies present in the $\alpha$ spectrum. Nevertheless, we may attempt to verify the hypothesis of the connection between the fundamental frequency and the flapwise mode, by analyzing the spectra of the oscillations of blade displacement 
in the rotor's axial direction, $u_{s t r_{x}}$. These out-of-rotor-plane displacements are strongly affected by flapwise deformation, but their dependence on torsion is minimal. Thus, by comparing the spectra of $u_{s t r_{h_{x}}}, \alpha$, and $\theta_{l L_{z}}$, we may elucidate the origin of the different frequencies present.

Figure 14 shows the spectra of $u_{s t r_{h_{x}}}$, and $\theta_{l L_{z}}$ for the same cases shown in figure 8 . Comparing the spectra for the cases of single-frequency oscillations (figures 8(a), 14(a), and 14(c)), we could see that the peak associated with the fundamental frequency in $\alpha$ also appears in the spectra of $u_{s t r_{h_{x}}}$, and $\theta_{l L_{z}}$. Nevertheless, the small traces of a secondary frequency that could be seen in $\alpha$ only appear in the spectrum of $\theta_{l L_{z}}$, and is absolutely absent in the spectrum of $u_{s t r_{h}}$. This seems to confirm that the fundamental frequency originates primarily in the flapwise motion. This observation becomes more evident when analyzing the case of multi-frequency oscillations (figures 8(b), 14(b), and 14(d)), where the peak of the fundamental frequency is much more prominent in the $u_{s t r_{h_{x}}}$ spectrum than in the $\theta_{l L_{z}}$ one. Contrarily, the same peaks of the secondary frequencies present in the $\alpha$ spectrum, appear prominently in the $\theta_{l L_{z}}$ spectrum, but are comparatively weak in the $u_{s t r_{h}}$ spectrum, which seems to indicate that those secondary frequencies originate from torsional deformation. This is consistent with the fact that the secondary frequencies are quasi-periodic in nature, and not just harmonics of the fundamental, as they originate from a different physical mechanism.

The oscillating transient behavior observed in this study suggest that the use of pitching as a short-term operational control method has several limitations which are inherent to the aeroelastic response of the rotor, which may preclude its usage as a means of mitigating the effects of rapid changes in the aerodynamic conditions on the blades such as the ones induced by tower interference, quick gusts, etc.

There is also the fact that rapid-pitching action may require substantially higher powers of actuation than the classical application of pitch as a method of power limitation for wind speeds above the nominal, due to the need of imposing a rapid angular acceleration to quickly turn the blade around its own axis. This aspect would become more critical as the size of the state-of-the art turbine increases. This upscaling in rotor size is a definitive trend for reduced cost-of-energy in the envisioned wind-power industry of the future. An increase in blade length results in a higher swept area, squaring the power generation but, there is also a cubical increase in the rotor weight as per the square-cube law (see [41]).

With the massive blades associated with the larger rotors to be used on future wind super turbines, it is necessary to use control systems which are less energy intensive than the pitch actuators. In this respect, innovative control methods such as active flow-control devices (FCDs) deserve to be studied in detail. FCDs are attachments that can be added to turbine blades capable of changing their aerodynamic characteristics. They are active control devices which require low-energy inputs to the actuating mechanisms, and that may respond rapidly to variations on the conditions occurring during turbine operation. Moreover, they could be used along with the classic pitch actuators, or with alternative control methods such as variable-speed stall control, and flexo-torsional adaptive blades, to create a hybrid low actuation energy blade that could eventually react fast enough to mitigate the effects of rapidly changing aerodynamic conditions. 
As an outlook for further work, we propose to continue this study with an analysis of the rotor aeroelastic response for a variation of the NREL-5MW RWT, which incorporates FCDs in the form of modular attachments based on the trailing-edge slotted-flap concept [42].

\section{Acknowledgments}

The authors gratefully acknowledge the financial support of National Science Foundation under grants CBET-0952218 and CMMI-1300970.

\section{References}

[1] EWEA, Upwind: Design limits and solutions for very large wind turbines, sixth Framework Programme, European Wind Energy Association, Brussels, Belgium, 2011.

[2] J. G. Stuart, A. D. Wright, C. P. Butterfield, Wind turbine control systems: dynamic model development using system identification and the FAST structural dynamics code, National Renewable Energy Laboratory, 1996.

[3] T. K. Barlas, G. Van Kuik, State of the art and prospectives of smart rotor control for wind turbines, in: Journal of Physics: Conference Series, vol. 75, IOP Publishing, 012080, 2007.

[4] J. W. V. Wingerden, A. W. Hulskamp, T. Barlas, B. Marrant, G. A. M. V. Kuik, D. P. Molenaar, M. Verhaegen, On the proof of concept of a 'smart'wind turbine rotor blade for load alleviation, Wind Energy 11 (3) (2008) 265-280.

[5] F. D. Bianchi, H. De Battista, R. J. Mantz, Wind turbine control systems: principles, modelling and gain scheduling design, Springer Science \& Business Media, 2006.

[6] E. Muljadi, K. Pierce, P. Migliore, Control strategy for variable-speed, stall-regulated wind turbines, in: Proceedings of the 1998 American Control Conference. ACC (IEEE Cat. No.98CH36207), vol. 3, ISSN 0743-1619, 1710-1714, 1998.

[7] E. Muljadi, C. P. Butterfield, Pitch-controlled variable-speed wind turbine generation, IEEE Transactions on Industry Applications 37 (1) (2001) 240-246.

[8] J. I. Herrera, T. W. Reddoch, J. S. Lawler, Harmonics generated by two variable speed wind generating systems, IEEE Transactions on Energy Conversion 3 (2) (1988) 267-273, ISSN 0885-8969.

[9] M. Steinbuch, Optimal multivariable control of a wind turbine with variable speed, Wind Engineering 11 (3) (1987) 153-163.

[10] H. Polinder, D. Bang, R. P. J. O. M. van Rooij, A. S. McDonald, M. A. Mueller, 10 MW Wind Turbine Direct-Drive Generator Design with Pitch or Active Speed Stall Control, in: 2007 IEEE International Electric Machines \& Drives Conference, vol. 2, 1390-1395, 2007.

[11] C. L. Bottasso, A. Croce, F. Gualdoni, P. Montinari, Load mitigation for wind turbines by a passive aeroelastic device, Journal of Wind Engineering and Industrial Aerodynamics 148 (2016) 57-69.

[12] Z. Salameh, Operation of the variable speed constant frequency double output induction generator (VSCF-DOIG) in a constant optimum power coefficient mode, Wind Engineering 9 (1985) 67-75.

[13] K. H. Hohenemser, A. H. P. Swift, On the Design of Horizontal Axis Two-Bladed Hinged Wind Turbines, Journal of Solar Energy Engineering 106 (2) (1984) 171-176.

[14] J. G. Njiri, D. Söffker, State-of-the-art in wind turbine control: Trends and challenges, Renewable and Sustainable Energy Reviews 60 (2016) 377-393.

[15] E. A. Bossanyi, Individual blade pitch control for load reduction, Wind Energy 6 (2) (2003) 119-128.

[16] E. A. Bossanyi, Further load reductions with individual pitch control, Wind Energy 8 (4) (2005) 481485.

[17] T. J. Larsen, H. A. Madsen, K. Thomsen, Active load reduction using individual pitch, based on local blade flow measurements, Wind Energy 8 (1) (2005) 67-80.

[18] K. A. Stol, W. Z. A. D. Wright, Individual blade pitch control for the controls advanced research turbine (CART), Journal of Solar Energy Engineering 128 (4) (2006) 498-505. 
[19] Z. Gao, X. Liu, M. Z. Q. Chen, Unknown Input Observer-Based Robust Fault Estimation for Systems Corrupted by Partially Decoupled Disturbances, IEEE Transactions on Industrial Electronics 63 (4) (2016) 2537-2547.

[20] X. Liu, Z. Gao, Robust finite-time fault estimation for stochastic nonlinear systems with Brownian motions, Journal of the Franklin Institute In Press.

[21] J. Chen, J. Pan, Z. Li, Y. Zi, X. Chen, Generator bearing fault diagnosis for wind turbine via empirical wavelet transform using measured vibration signals, Renewable Energy 89 (2016) 80 - 92.

[22] I. V. deBessa, R. M. Palhares, M. F. S. V. D'Angelo, J. E. C. Filho, Data-driven fault detection and isolation scheme for a wind turbine benchmark, Renewable Energy 87, Part 1 (2016) $634-645$.

[23] P. F. Odgaard, J. Stoustrup, M. Kinnaert, Fault Tolerant Control of Wind Turbines - a benchmark model, IFAC Proceedings Volumes 42 (8) (2009) 155 - 160.

[24] Y. Vidal, C. Tutivn, J. Rodellar, L. Acho, Fault Diagnosis and Fault-Tolerant Control of Wind Turbines via a Discrete Time Controller with a Disturbance Compensator, Energies 8 (5) (2015) 4300.

[25] P. F. Odgaard, J. Stoustrup, M. Kinnaert, Fault-Tolerant Control of Wind Turbines: A Benchmark Model, IEEE Transactions on Control Systems Technology 21 (4) (2013) 1168-1182.

[26] P. F. Odgaard, K. E. Johnson, Wind turbine fault detection and fault tolerant control - An enhanced benchmark challenge, in: 2013 American Control Conference, 4447-4452, 2013.

[27] W. Chen, S. Ding, A. Haghani, A. Naik, A. Khan, S. Yin, Observer-based FDI Schemes for Wind Turbine Benchmark, IFAC Proceedings Volumes 44 (1) (2011) 7073 - 7078, 18th IFAC World Congress.

[28] R. Chaaban, D. Ginsberg, C. P. Fritzen, Structural Load Analysis of Floating Wind Turbines Under Blade Pitch System Faults, in: N. Luo, Y. Vidal, L. Acho (Eds.), Wind Turbine Control and Monitoring, Springer, Zurich, Switzerland, ISBN 978-3-319-08413-8, 301-334, 2014.

[29] J. Jonkman, S. Butterfield, W. Musial, G. Scott, Definition of a 5-MW reference wind turbine for offshore system development, Tech. Rep. NREL/TP-500-38060, National Renewable Energy Laboratory, 2009.

[30] F. L. Ponta, A. D. Otero, L. I. Lago, A. Rajan, Effects of rotor deformation in wind-turbine performance: The Dynamic Rotor Deformation Blade Element Momentum model (DRD-BEM), Renewable Energy 92 (2016) 157-170.

[31] A. D. Otero, F. L. Ponta, Structural Analysis of Wind-Turbine Blades by a Generalized Timoshenko Beam Model, Journal of Solar Energy Engineering 132 (1) 011015, ISSN 0199-6231.

[32] W. Yu, D. H. Hodges, V. Volovoi, C. E. S. Cesnik, On Timoshenko-like modeling of initially curved and twisted composite beams, Int. J. Sol. and Struct. 39 (2002) 5101-5121.

[33] D. H. Hodges, Nonlinear Composite Beam Theory, AIAA, Reston, Virginia, 2006.

[34] W. Xudong, W. Z. Shen, W. J. Zhu, J. N. Srensen, C. Jin, Shape optimization of wind turbine blades, Wind Energy 12 (8) (2009) 781-803.

[35] J. F. Manwell, J. G. McGowan, A. L. Rogers, Wind energy explained: Theory, design and application, Wiley, Chichester, UK, 2002.

[36] T. Burton, D. Sharpe, N. Jenkins, E. Bossanyi, Wind Energy Handbook, Wiley, Chichester, UK, 2001.

[37] IEC, Wind Turbine Generator Systems - Part 13: Measurement of mechanical loads, Report IEC/TS 61400-13, International Electrotechnical Commission (IEC), 2001.

[38] P. Jamieson, Innovation in wind turbine design, Wiley, 2011.

[39] C. Crawford, Re-examining the precepts of the blade element momentum theory for coning rotors, Wind Energy 9 (5) (2006) 457-478.

[40] C. Crawford, J. Platts, Updating and optimization of a coning rotor concept, Journal of Solar Energy Engineering 130 (2008) 031002.

[41] R. Thresher, S. Schreck, M. Robinson, P. Veers, Wind energy status and future wind engineering challenges, in: Proceedings of the 1st American Association for Wind Engineering Workshop. Vail, Colorado (USA), NREL/CP-500-43799, 2008.

[42] M. Menon, F. Ponta, X. Sun, Q. Dai, Aerodynamic Analysis of Flow-Control Devices for Wind Turbine Applications Based on the Trailing-Edge Slotted-Flap Concept, Journal of Aerospace Engineering $29(5)$. 